\title{
Malignant Peripheral Nerve Sheath Tumor of the Infraorbital Nerve
}

\author{
José Luis D’Addino ${ }^{1}$ Laura Piccoletti ${ }^{2}$ María Mercedes Pigni ${ }^{3}$ Maria José Rodriguez Arenas de Gordon ${ }^{3}$
}

1 General Surgery and Head and Neck Division, Vicente López Public

Address for correspondence José Luis D’Addino, Vicente López Public Hospital, Buenos Aires, Argentina

2 Division of General Surgery, Vicente López Public Hospital, Buenos Aires, Argentina

${ }^{3}$ Division of Head and Neck Surgery, Vicente López Public Hospital, Hospital, Adolfo Alsina 1991, Florida (1602). Buenos Aires, Argentina (e-mail: jldaddino@gmail.com).

Buenos Aires, Argentina

Craniomaxillofac Trauma Reconstruction 2016;9:170-174

\begin{abstract}
The objective of this study is to report a large, rare, and ulcerative infiltrated skin lesion. Its diagnosis, therapeutic management, and progress are described. The patient is a 78-year-old white man, who presented with a 12-month ulcerative perforated lesion that had affected and infiltrated the skin, with easy bleeding. He had a history of hypertension, although controlled, was a 40-year smoker, had chronic atrial fibrillation, diabetes, and microangiopathy. During the consultation, the patient also presented with ocular obstruction due to an inability to open the eye. He mentioned having reduced vision. The computed tomography scan showed upper maxilla osteolysis without eye involvement. We underwent a radical resection in which upper maxilla and the anterior orbital

Keywords

- schwannoma

- peripheral nerve sheath tumor

- malignant schwannoma

- cheek reconstruction margin were included. We used a Becker-type flap that allowed us to rebuild the cheek and to complete a modified neck dissection. Progress was favorable; the patient recovered ocular motility and his vision improved to 20/200. The final biopsy result was "malignant peripheral nerve sheath tumor, malignant schwannoma." Malignant schwannoma of the peripheral nerve is extremely rare. The total resection and reconstruction being completed in one surgery represented a challenge due to the difficulty in obtaining tissues in addition to the necessity of an oncological resection.
\end{abstract}

Peripheral nerve sheath malignant tumors, also known as fusiform cells/Schwann cell malignant tumors, neurofibrosarcoma, or neurogenic sarcoma, represent $10 \%$ of soft tissue sarcomas, and only 8 to $16 \%$ of them are located in head and neck. $^{1,2}$

Malignant peripheral nerve cell tumor or malignant neurilemmoma is a form of cancer of the connective tissues surrounding nerves. It is classified as a sarcoma because of its origin and behavior. About half cases are diagnosed in people with neurofibromatosis; the lifetime risk for a malignant peripheral nerve cell tumor in patients with neurofibromatosis type I is 8 to $13 \%{ }^{3}$ This tumor and the rhabdomyoblastomatosis component are called malignant triton tumors. The diagnosis of malignant peripheral nerve

received

December 9, 2014

accepted after revision

June 14, 2015

published online

November 13, 2015

cell tumor has also been complicated by unclear criteria for determining the malignancy of a tumor that has originated in the nerve. Since Harkin et al (1978) first reported plexiform schwannoma, ${ }^{4-6}$ several authors have reported plexiform schwannoma unassociated with Von Reckling Hausen disease, which usually show histological features. ${ }^{7,8}$

The case presented herein is extremely rare as it involves the infraorbital nerve with both bone and skin infiltration. We describe its management and final progression.

\section{Clinical Report}

A 78-year-old man is referred to our head and neck section in light of a 12-month history of a rapidly growing

Copyright $\odot 2016$ by Thieme Medical Publishers, Inc., 333 Seventh Avenue, New York, NY 10001, USA. Tel: +1(212) 584-4662.
DOI http://dx.doi.org/ $10.1055 / \mathrm{s}-0035-1563698$ ISSN $1943-3875$. 


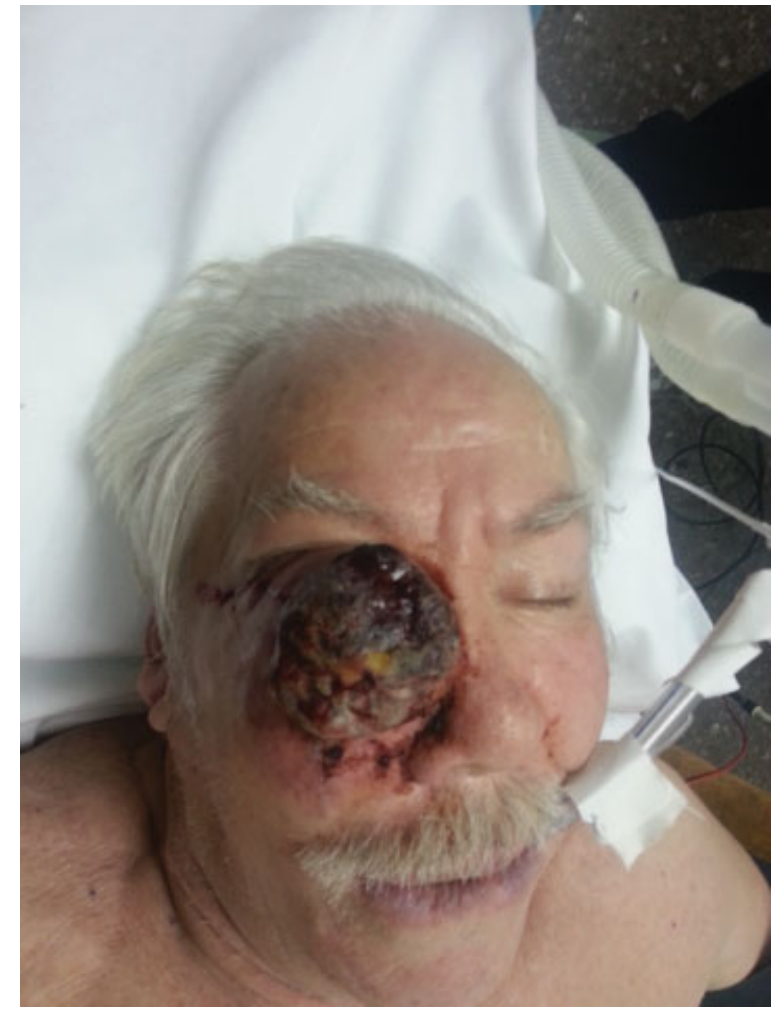

Fig. 1 Exophytic lesion of right cheek.

ulcerovegetant lesion (approximate size $10 \times 10 \mathrm{~cm}$ ) located in the right cheek. The lesion was of relatively large breadth, extending to the inferior eyelid causing homolateral eye occlusion. It was prone to recurrent bleeding, rigid, and fixed to the maxilla and the ocular orbital. It originally started as a hard, painless, subcutaneous lump in the cheek, which later developed hypoesthesia, ulceration, and recurrent episodes of profuse bleeding, which responded to compression (-Fig. 1). Comorbidities included: 40 years of smoking, hypertension-on enalapril, chronic atrial fibrillation-on aspirine (AAS), type 2 diabetes-on metformin, diabetic neuropathy, and microangiopathy. There was no personal or family history of neurofibromatosis I or Von Recklinghausen-related illnesses. The clinical examination showed loss of visual acuity (Snellen test 20/200) and normal eye move-

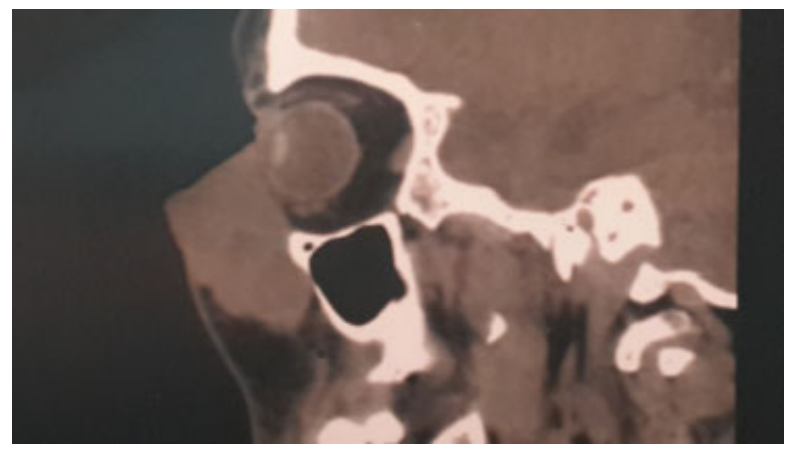

Fig. 2 Preoperative CT scan.

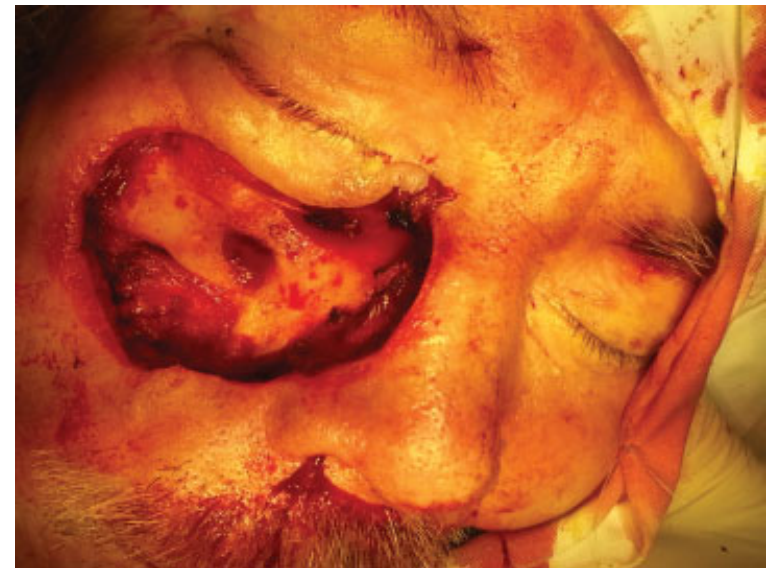

Fig. 3 Resection of the lesion and maxillary hole.

ments; however, the eye had been occluded for over a year due to tumoral compression. Two palpable nodules were evident in the neck, located at level II.

A computed tomography scan was requested and it showed (-Fig. 2) "a process of the maxilla involving the infraorbital foramen and superior maxilla bone. Both orbit and the eyeball were tumor free. Enlarged right laterocervical lymphadenopathies." Routine preoperative tests were normal, although for such an extensive surgery, his comorbidities did put him at an extremely high cardiac risk. However, surgical intervention was decided upon. It was a 2.5-hour procedure, under general anesthesia, which involved a wide

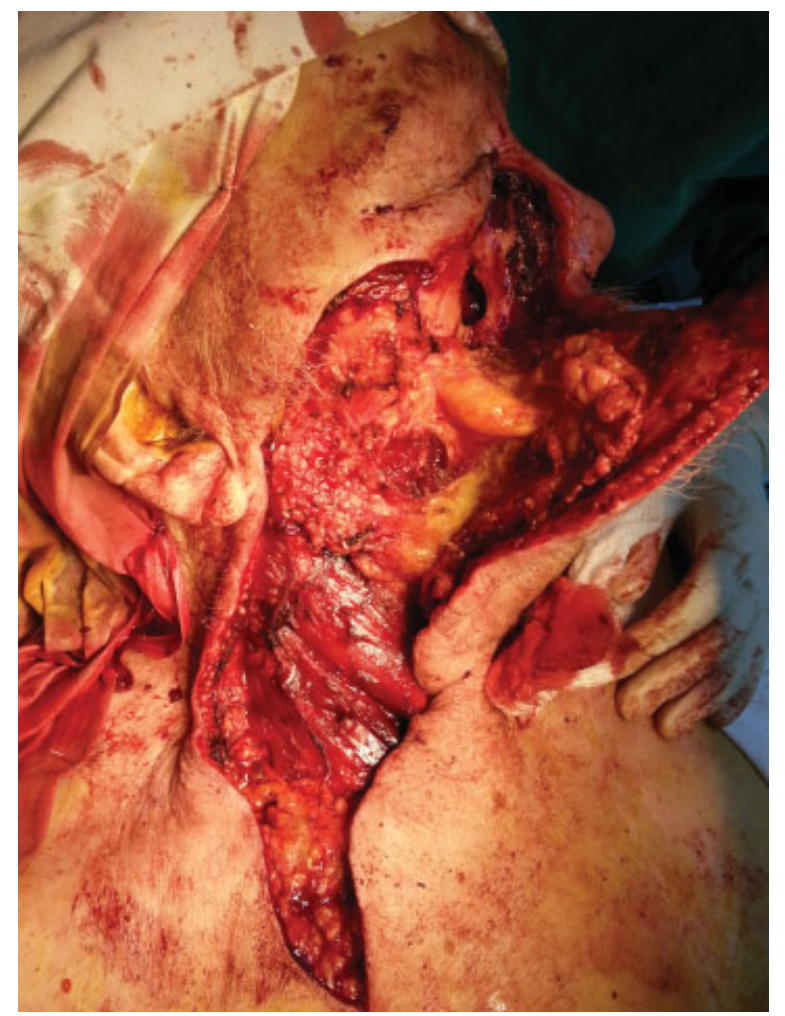

Fig. 4 Mobilized flap and neck dissection. 


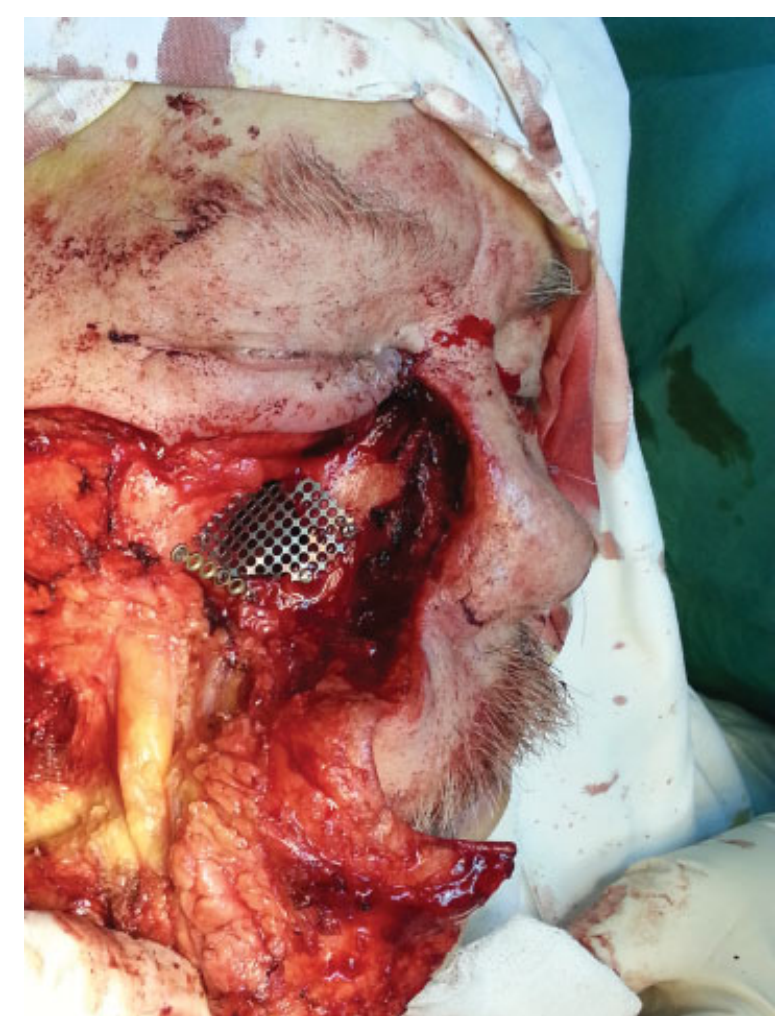

Fig. 5 Fixation of titanium plate and mesh.

resection partially including the maxilla as well as the inferior orbital rim with freezing biopsy margin control. We conducted a biopsy freezing the borders to ensure their being negative at both the bony and cutaneous levels, by macroscopic and histology study of the sample (-Fig. 3). Bone reconstruction was done by screwing in titanium plates and mesh with titanium screws. A Becker-type cervicopectoral graft was performed from the chest, and a modified cervical lymphadenectomy, that included levels II to IV, was undertaken through the same incision. The skin flap achieved complete cover of the defect (-Figs. 4 and $\mathbf{5}$ ). He had a good postoperative recovery and was discharged 48 hours later. We were able to successfully partially preserve the inferior eyelid, and by freeing the eyeball, visual acuity in the affected eye improved to $20 / 100$.

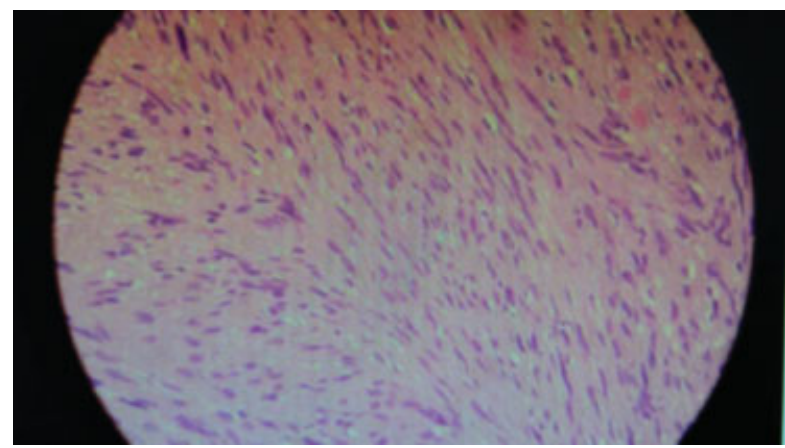

Fig. $6 \mathrm{H} / \mathrm{E} \times 400$. Fusocellular proliferation.

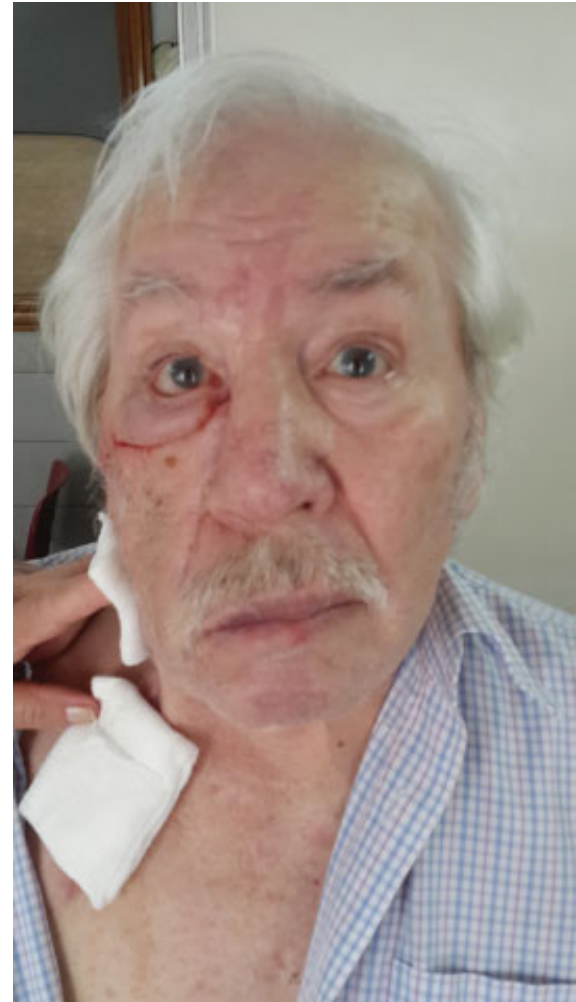

Fig. 7 One month postoperatively.

Biopsy results showed atypical fusocellular proliferation, which required immunostaining: vimentin positive, S100 positive, AE1-AE3 negative, and AM1 negative (-Fig. 6). Cytology and immunophenotype findings were compatible with malignant peripheral nerve tumor: malignant schwannoma with bone infiltration. Ten of the resected cervical lymph nodes were reactive. No postoperative complications occurred, the only concern was aesthetic, due to sectoral peripheral paralysis of the facial nerve due to partial section of cervicofacial branches in the dissection of the flap ( - Fig. 7). The area was treated by oncology with chemotherapy via doxorubicin and remained clear of illness for approximately 1 year (-Fig. 8). He later developed bilateral pulmonary "cannonball metastases" without relapse of the initial area of occurrence. He passed away

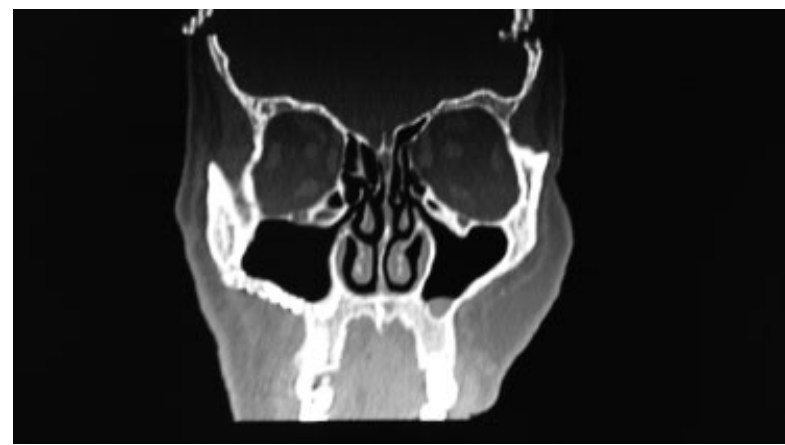

Fig. 8 Postoperative CT scan. 


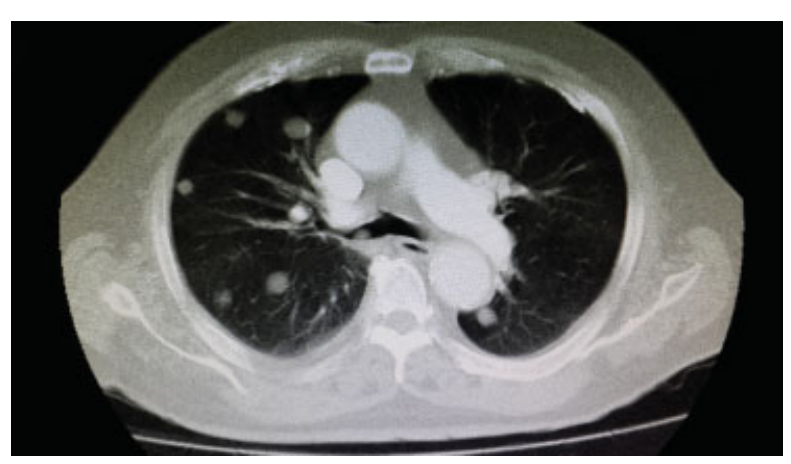

Fig. 9 Lung metastasis. CT scan.

approximately 18 months from the initial date of surgery, due to his pulmonary complications (-Fig. 9).

\section{Discussion}

The Schwann cells give rise to neurofibroma and neurilemmoma (schwannoma). A schwannoma is a slow-growing, solitary, and encapsulated tumor attached to a nerve. This type of tumor may arise from any cranial or spinal nerve that has a sheath, or any motor or sensory nerve other than optic and the olfactory nerves, which do not have a Schwann cell sheath. Schwannoma was first established as a pathological entity described by Verocay in $1908 .{ }^{9}$ A malignant peripheral nerve tumor is a type of sarcoma; half of the cases are diagnosed in patients who suffer from neurofibromatosis. The risk of developing a malignant peripheral nerve sheath sarcoma in patients with neurofibromatosis type I is around $10 \%$. The denomination malignant peripheral nerve sheath tumor is named by the World Health Organization's classification. It accounts for $10 \%$ of soft tissue sarcomas, its location in head and neck being between 8 and 16\%. Despite being extremely rare, it has been described in other bodily locations and occasionally associated to neurofibromatosis type I or Von Recklinghausen disease. ${ }^{1,10,11}$ It affects mainly patients between 20 and 50 years old with no gender preference. Sporadic malignant schwannomas publications, which are even more infrequent, have been described in locations such as orbit, neck, and parapharyngeal region. ${ }^{1}$ From the sources detailed in the bibliography, bone and skin involvement are very unusual, which is what makes this case so exceptional, seeing that the tumor presented was associated with osteolysis of the maxilla as well as skin ulceration.

In general, these tumors appear as circumscript submucous lumps with associated pain or paresthesia, occasionally muscular weakness and atrophy. ${ }^{12}$ They are always aggressive and grow quickly. ${ }^{1}$ They can affect the bone, which they initially erode and later infiltrate their bone marrow. The affection of the bone may have three different mechanisms: (1) erosion into the bone secondarily, (2) it can develop within a nutrient canal primarily, involving the bone secondarily, and (3) primarily arise within central medullary canal. These sarcomas disseminate initially by contiguity and later by bloodstream or along the sheath of the affected nerve. Lymph node metastases are infrequent; however, bloodstream dissemination is present in $50 \%$ of cases. ${ }^{13,14}$ Our patient did not present lymph node metastasis.

The final diagnosis for malignant peripheral nerve cell tumor is histopathological. Immunohistochemistry plays an important part in the diagnosis and differential diagnosis, excluding fibrosarcoma, synovial sarcoma, fibrous histiocytoma, adenoid cystic carcinoma, neurogenic sarcoma, and chondrosarcoma. The tumor cells show immune reactivity for the $\mathrm{S} 100$ protein and vimentin with focal positivity to CD 68 and negativity to Keratin.

On a CT scan, it presents a hypodense, nonhomogeneous mass due to areas of degeneration and areas of varying cellular density and osteolysis.

The elective treatment is radical extirpation with wide and safety margin to reduce local recurrence. ${ }^{15-17}$ In this patient, due to his elevated cardiac surgical risk and associated vasculopathy, we chose to use a Becker-type cervicopectoral graft irrigated by perforating arteries from the internal mammary artery which not only provided very good circulation but simultaneously reduced the operative time when compared with using a microvascular graft. This musculocutaneous graft proved an adequate size both to cover the maxillary reconstruction prosthesis as well as allowing being performed the lymphadenectomy. Keeping in mind his high cardiovascular risk, this surgical plan made it possible to perform the oncological resection, removal, and reconstruction all-in-one short operating period. Adjuvant treatments such as chemotherapy (doxorubicin) and radiotherapy are controversial. $^{1-3,18-20}$ Our patient received postoperative chemotherapy without neither evidence of relapse in the area nor systemic illness during a period of 1 year (clinical and CT scan follow-up). At the end of said time frame, he developed bilateral pulmonary metastases, the complications of which lead to his death 6 months later. The patient's survival is correlated to the size of lesion, adequacy of margins, and whether or not there is an association with neurofibromatosis type I as well as immunohistochemical findings. The overall survival rate is 40 to $70 \%$ with a poor prognosis. $^{21}$

\section{Conclusion}

Malignant schwannoma is an aggressive tumor with a difficult treatment. Early detection can aid in reducing its morbidity; however, its dissemination determines its bad prognosis.

Although chemo- and radiotherapy are often indicated, there is still insufficient statistical data to warrant their use, making the initial/early wide resection the recommended treatment. Chemotherapy with a high dose of doxorubicin and oftentimes radiotherapy are done as adjuvant/neoadjuvant treatments, but their role is questionable.

Conflict of Interest

None. 


\section{References}

1 McCarthy JosephG. Cirugía Plástica. La cara. II. Panamericana Editorial 1992;12:1111-1158

2 Mallick S, Gandhi AK, Joshi NP, et al. Re-irradiation in head and neck cancers: an Indian tertiary cancer centre experience. The Journal of Laryngology \& Otology 2014;128(11):996-1002

3 Lee JH, Lee HK, Chui CG. Malignant peripheral nerve sheath tumor in paraphaaryngeal space-tumor spread through Eustachian tube. ANNR Am J Neuroradiol 2001;2(4):748-750

4 Maranjan SN, Jagasia V, Pusalkar A. Schwannoma of the cheek. Indian J Otolaryngol Head and Neck Surg 2001;53(2):140-141

5 Hasturk AE, Basmaci M, Bayram C, Bozdogan N. Surgical management of recurrente malignant schwannoma of the scalp. J Craniofac Surg 2011;22(3):1120-1122

6 Dijkstra MD, Balm AJM, Gregor RT, Hilgers FJM, Lofrus BM. Soft tissue sarcomas of the head and neck associated with surgical trauma. The Journal of Laryngology \& Otology 1995; 109(021995:126-129

7 Endo M, Yamamoto H, Harimaya K, Kohashi K, Ishii T, Setsu N, Iwamoto Y, Oda Y. Hum Patho 2013;44(12):2845-2848

8 Hilton DA, Hanemann CO. Schwannomas and their pathogenesis. Brain Pathol 2014;23:235-246

9 Bagan JV, Sanchis JM, et al. Malignant peripheral nerve sheath tumor of the maxilla. A case report. Oral Oncology Extra 2005; 41(4):70-73

10 Wiesmiller K, Barth TFE, Gronau S. Early radiation-induced malignant fibrous histiocytoma of the oral cavity. J Laryngol Otol 2003; 117(3):224-226

11 Bullock MJ, Bedard YC, Bell RS, Kandel R. Intraosseous MPNST. Case report and review of literature. Ach Pathol Lab Med 2006;119(4): 367-370

12 Dinesh Kumar Sharma, Barjinder Singh Sohal, T.L. Parmar, and Harvinder Arora. Schwannomas of head and neck and review of literature. Indian J Otolaryngol Head Neck Surg 2012;64(2): 177-180

13 Sham M.E. Ghorpade, Akshya Shetty, Sri Hari, Vinay. Malignant peripheral nerve cell tumor. J Maxillofac Oral Surg 2010;9(1): 68-71

14 Harkin JC, Arrington JH, Reed RJ. Benign plexiform schwanomma a lesion descrete from plexiform neurofibroma. J Neuropath Exp Neurol 1978;37:622

15 Kerstin Wiesmiller, Thomas F. E. Barth and Silke Gronau: Early radiation-induced malignant fibrous histiocytoma of the oral cavity. The Journal of Laryngology \& Otology 2003;117(3): 224-226

16 N Dawe, J Patterson, D Hamilton and C Hartley: Targeted use of endoscopic $\mathrm{CO} 2$ laser cricopharyngeal myotomy for improving swallowing function following head and neck cancer treatment. The Journal of Laryngology \& Otology 2014;128(12): $1105-1110$

17 C Williams, R Byrne, D Holden, I Sherman and V R Srinivasan: Twoweek referrals for suspected head and neck cancer: two cycles of audit, 10 years apart, in a district general hospital. The Journal of Laryngology \& Otology 2014;128(8):72

18 González OrpusAlvarez Morujo R, García LealR, Lasso VázquezJM, Scpña YurrotaB. Malignanr peripheral nerve sheath tumor of the infra-orbital nerve. Article in Spanish Neurocirugia (astur) 2014; 25(5):240-243

19 Tezer MS, Ozcan M, Han O, Unal A, Ozlugedik S. Schwannoma originating from the infraorbital nerve: a case report. Auris Nasus Larynx 2006;33(3):343-345

20 Sierszen W, Stankiewicz C. Malignant schwannoma of infraorbital nerve. Otolaryngol Pol 2003;57(4):573-576

21 Lyons CJ, McNab AA, Garner A, Wright JE. Orbital malignant peripheral nerve sheath tumours. Br J Ophthalmol 1989;73(9): 731-738 